\title{
INFLUÊNCIA DA CONCENTRAÇÃO DE POLPA DE GOIABA NA ACEITAÇÃO DE FERMENTADO DE KEFIR
}

\section{Influence of the guava pulp concentration on fermented kefir acceptance}

\author{
Nkarthe Guerra Araújo ${ }^{1^{*}}$, José Barros da Silva ${ }^{2}$, Idiana Macêdo Barbosa ${ }^{2}$, \\ Cláudia Souza Macêdo ${ }^{2}$
}

\begin{abstract}
RESUMO
Este trabalho teve por objetivo desenvolver e avaliar a aceitação e intenção de compra de fermentado de kefir saborizado com diferentes concentrações de polpa de goiaba pasteurizada. Para sua elaboração, o leite in natura foi adicionado de $2 \%$ de leite em pó e em seguida, submetido ao tratamento térmico a $90{ }^{\circ} \mathrm{C} / 3 \mathrm{~min}$ e resfriado a $25^{\circ} \mathrm{C}$, etapa esta em que foi inoculado $3 \%$ dos grãos de kefir. A fermentação ocorreu à temperatura ambiente a $25 \pm 2{ }^{\circ} \mathrm{C} / 24 \mathrm{~h}$. Após recuperação dos grãos, o kefir foi armazenado a $5{ }^{\circ} \mathrm{C} / 24 \mathrm{~h}$ e em seguida foram estabelecidas quatro formulações distintas: K0: kefir natural com $12 \%$ de sacarose sem adição de polpa de goiaba, K1, K2 e K3: kefir saborizado com $8 \%$, 10\% e 12\% de polpa de goiaba $+12 \%$ de sacarose, respectivamente. As formulações desenvolvidas apresentaram valores de $\mathrm{pH}$ variando de 3,5 a 3,7 e acidez titulável com variações de 1,2 a 1,5\% de ácido lático. Verificou-se que houve diferença significativa $(\mathrm{p}<0,05)$ para os atributos cor, aroma, sabor e aceitação global das amostras K0, K1 e K2 em relação a K3, bem como, da intenção de compra. Entretanto, o atributo consistência não foi significativamente afetado. A formulação K3, com $12 \%$ de polpa de goiaba apresentou melhores médias para todos os atributos analisados (cor: 8,5, sabor: 7,7, aroma: 7,4 e aceitação global: 7,7) e melhor intenção quanto à compra $(4,3)$, portanto, nas condições propostas é a concentração mais indicada para sua saborização.
\end{abstract}

Palavras-chave: análise sensorial; leite fermentado; Psidium guajava.

1 Universidade Federal da Paraíba (UFPB), Cidade Universitária, 58051-900, João Pessoa, PB, Brasil. E-mail: nkarthe@gmail.com

2 Universidade Federal do Rio Grande do Norte (UFRN), Natal, RN, Brasil.

* Autor para correspondência.

Recebido / Received: 18/12/2016

Aprovado / Approved: 01/02/2018 


\begin{abstract}
This work aimed to develop and evaluate the acceptance and intention to buy fermented kefir flavored with different concentrations of pasteurized guava pulp. For its preparation, in the raw milk was added $2 \%$ of milk powder and then subjected to heat treatment at $90{ }^{\circ} \mathrm{C} / 3 \mathrm{~min}$ and cooled to $25{ }^{\circ} \mathrm{C}$, in this stage $3 \%$ of kefir beans were inoculated. The fermentation occurred at room temperature $\left(25 \pm 2{ }^{\circ} \mathrm{C} / 24 \mathrm{~h}\right)$. After recovery of the grains, the kefir was stored at $5{ }^{\circ} \mathrm{C} / 24 \mathrm{~h}$ and then four different formulations were made: K0-natural kefir with $12 \%$ sucrose without addition of guava pulp, K1, K2 and K3-kefir flavored with $8 \%, 10 \%$ and $12 \%$ guava pulp $+12 \%$ sucrose, respectively. The developed formulations had $\mathrm{pH}$ values ranging from 3.5 to 3.7 and titratable acidity with variations of 1.2 to $1.5 \%$ lactic acid. It was verified that there was a significant difference $(\mathrm{p}<0.05)$ for the attributes color, aroma, flavor and overall acceptance of samples K0, K1 and K2 in relation to $\mathrm{K} 3$, as well as the intention to buy. However, the attribute consistency was not significantly affected. The K3 formulation, with $12 \%$ of guava pulp showed better means for all attributes analyzed (color: 8.5, flavor: 7.7, aroma: 7.4 and overall acceptance: 7.7) and better intention to buy (4.3), therefore, under the proposed conditions, it is the most indicated concentration for fermented kefir flavoring.
\end{abstract}

Keywords: Sensory analysis; fermented milk; Psidium guajava.

\section{INTRODUÇÃO}

Kefir é o produto obtido pela fermentação do leite e/ou leite reconstituído com micro-organismos específicos, podendo ser adicionado ou não de outras substâncias alimentícias. Tais micro-organismos são compostos por uma combinação de bactérias e leveduras, como Lactobacillus kefir, espé cies dos gêneros Leuconostoc, Lactococcus e Acetobacter, Lactobacillus casei, Bifidobaterium sp. e Streptococcus salivarius subsp. thermophilus, além de leveduras fermentadoras (Kluyveromyces marxianus) e não fermentadoras de lactose (Saccharomyces omnisporus, Saccharomyces cerevisae e Saccharomyces exiguus), que atuam em simbiose (BRASIL, 2007), e por sua ação, conferem a este produto diversos efeitos benéficos.

O kefir é indicado na prevenção de uma variedade de patologias, incluindo doenças metabólicas, alérgicas e aterosclerose, câncer e distúrbios gastrintestinais. Seu consumo é estimulado ainda para pacientes com intolerância a lactose, sendo considerado também como fortalecedor do sistema imunológico (DALLA SANTA et al., 2008). Algumas propriedades antitumorais, antibacterianas e antifúngicas foram demonstradas em pesquisas in vitro e com animais de laboratório (MONTANUCI et al., 2010). Diante dos benefícios que esse alimento pode proporcionar à saúde, torna-se fundamental a busca e o desenvolvimento de novas formulações. Entretanto, pesquisas relacionadas ao perfil sensorial, utilização e desenvolvimento de novos produtos com kefir ainda são escassos (SANTOS; BASSO, 2013).

A goiaba (Psidium guajava) destaca-se como uma excelente fonte de vitamina $\mathrm{C}$, além disto, contém quantidades razoáveis de pró-vitamina A e do complexo B e minerais como cálcio e fósforo (FERNANDES et al., 2009). Apresenta, ainda, açúcares e pectina, taninos, flavonóides, óleos essenciais, alcoóis 
sesquiterpenóides e ácidos triterpenóides. Muitos destes compostos exibem propriedade antioxidante, prevenindo doenças crônicas degenerativas como o câncer (NASCIMENTO et al., 2010). É considerada um dos frutos de maior importância nas regiões tropicais e subtropicais, não apenas pelo valor nutritivo, mas também pela excelente aceitação para consumo in natura e pela grande aplicação industrial (SILVA et al., 2010). Neste sentido, o processamento para obtenção de polpa é uma atividade agroindustrial significativa, à medida que agrega valor econômico à fruta, evitando desperdícios e minimizando perdas, que podem ocorrer durante a comercialização do produto in natura (EVANGELISTA; VIEITES 2006).

Considerando tais aspectos, este trabalho teve por objetivo desenvolver e avaliar a aceitação e intenção de compra de fermentado de kefir saborizado com diferentes concentrações de polpa de goiaba pasteurizada, bem como, determinar a formulação mais aceita.

\section{MATERIAL E MÉTODOS}

\section{Obtenção da polpa de goiaba pasteurizada}

Os frutos e o leite in natura foram adquiridos em propriedade rural no município de Macaíba, localizado na região metropolitana de Natal-RN. Para obtenção da polpa, $2 \mathrm{~kg}$ de goiaba da variedade Paluma em estágio fisiológico de maturação completo, selecionadas pela ausência de injúrias, foram lavadas em água corrente, sanitizadas em água clorada a 30 ppm por 15 min e secas naturalmente. Posteriormente, foram fracionadas com casca em pequenas fatias e trituradas em multiprocessador até obtenção de uma polpa homogênea, que foi transferida para um filtro de tela em nylon, onde foram removidos os resíduos (cascas e sementes). O rendimento de polpa resultou em $82 \%$, teor de sólidos solúveis igual a 9, $4^{\circ}$ Brix, $\mathrm{pH} 3,7$ e acidez titulável de $0,99 \%$. A polpa obtida foi adicionada de $12 \%$ de sacarose (porcentagem calculada em relação à quantidade de fermentado de kefir elaborado) e em seguida, pasteurizada a $85^{\circ} \mathrm{C} / 5 \mathrm{~s}$ e resfriada a $10 \pm 1{ }^{\circ} \mathrm{C}$.

\section{Fermentação e saborização do kefir}

Oito litros de leite com acidez titulável de $0,17 \mathrm{~g}$ ácido lático/ $100 \mathrm{~mL}, \mathrm{pH} 6,6,3,7 \%$ de gordura, $3,1 \%$ de proteínas, $8,6 \%$ de extrato seco desengordurado, densidade $1,031 \mathrm{~g} / \mathrm{mL}$, ponto de crioscopia $-0,512^{\circ} \mathrm{C}$ e extrato seco total $12,3 \%$, foram adicionados de $2 \%$ de leite em pó, submetidos ao tratamento térmico a $90{ }^{\circ} \mathrm{C} / 3$ min e posteriormente resfriados a $25{ }^{\circ} \mathrm{C}$, etapa esta na qual foi inoculado $3 \%$ dos grãos de kefir. A fermentação ocorreu à temperatura ambiente $\left(25 \pm 2{ }^{\circ} \mathrm{C}\right)$, por $24 \mathrm{~h}$. Em seguida, os grãos foram recuperados com auxilio de uma espátulade poliestireno e filtro de tela em nylon. O leite fermentado obtido foi então armazenado sobre refrigeração a $5 \pm 1{ }^{\circ} \mathrm{C} / 24 \mathrm{~h}$. Após este período, foram definidas quatro formulações distintas: K0 (kefir natural sem adição de polpa e com $12 \%$ de sacarose) K1, K2 e K3, (kefir saborizado com $8 \%, 10 \%$ e $12 \%$ de polpa de goiaba previamente pasteurizada com $12 \%$ de sacarose, respectivamente). As diferentes formulações foram armazenadas em garrafas de poliestireno sob refrigeração a $5 \pm 1{ }^{\circ} \mathrm{C} /$ $24 \mathrm{~h}$ para incorporação do sabor e análises posteriores.

\section{Análises sensoriais e físico-químicas}

Aplicou-se um questionário relacionado ao hábito de consumo de kefir, e posteriormente, uma análise de aceitação e intenção de compra com 50 julgadores não treinados. Antes da execução da avaliação sensorial, foi submetido e aprovado um Projeto pelo Comitê de Ética em Pesquisa da 
Universidade Federal do Rio Grande do Norte, CEP/UFRN, (Parecer CEP $\mathrm{n}^{\mathrm{0}} 1.808 .108$, CAAE - 58886016.0.0000.5537).

A intenção de compra foi avaliada por meio de escala de cinco pontos $(5=$ certamente compraria; 1 = certamente não compraria) e o grau de aceitação por meio de teste afetivo laboratorial com uso de escala hedônica de nove pontos $(9=$ gostei muitíssimo, 1 = desgostei muitíssimo) para os atributos, cor, aroma, consistência, sabor e aceitação global (MEILGAARD et al., 1991). As análises foram realizadas três dias após a elaboração do fermentado com estudantes e servidores da Escola Agrícola de Jundiaí (EAJ/UFRN), na faixa etária variando de 18 a 44 anos. Para ambos os testes sensoriais, cerca de $30 \mathrm{~mL}$ de fermentado de kefir foi servido em copos descartáveis de $50 \mathrm{~mL}$, codificados com números aleatórios de três dígitos. Para eliminar o sabor residual entre as amostras, foram oferecidos biscoitos tipo cream cracker e água mineral. Foram realizadas também, após fermentação por 24h, análises de $\mathrm{pH}$ e acidez titulável nas formulações desenvolvidas, conforme metodologia proposta pela Association Official Analytical Chemists (AOAC, 2000).

\section{Análise estatística dos dados}

Os resultados obtidos foram avaliados pelo delineamento inteiramente casualizado (DIC) e interpretados pela análise de variância
(ANOVA) e comparação de médias pelo Teste de Tukey ao nível de 5\% de significância, utilizando-se o Programa Assistat 7.7. Para avaliar a interação entre os atributos cor, aroma, consistência e sabor, foi realizada uma análise Multivariada e teste de Componentes Principais (PCA), utilizando-se o Software ActionStat 3.3. O atributo aceitação global foi omitido para adequação do tratamento estatístico.

\section{RESULTADOS E DISCUSSÃO}

As formulações de fermentado de kefir não diferiram significativamente $(\mathrm{p}>0,05)$ com relação aos valores de acidez titulável (1,2 a 1,5\%) e pH (3,5 a 3,7), Tabela 1 . A acidez titulável atende o estabelecido pela legislação, que recomenda teores entre 0,5 a 1,5 g.100 g-1 de ácido láctico, para este tipo de produto (BRASIL, 2000). As formulações elaboradas com 8,10 e $12 \%$ de polpa de goiaba apresentaram valores de $\mathrm{pH}$ ligeiramente inferior e acidez titulável superior a formulação de fermentado de kefir sem adição da polpa. Isso deve estar relacionado com os teores de ácidos orgânicos das frutas, presentes nas mesmas, conforme observado por Dalla Santa et al. (2008), que avaliaram o kefir natural e saborizado com polpa de ameixa e morango, obtendo resultados de $4,6,4,1$ e 4,2 para o $\mathrm{pH}$ e $0,62,0,79$ e $0,67 \%$ de acidez titulável, respectivamente. Weschenfelder et al. (2011) avaliaram duas

Tabela 1 - Acidez titulável e pH de fermentado de kefir natural e saborizado com diferentes concentrações de polpa de goiaba

\begin{tabular}{ccccc}
\hline Análises & $\mathrm{K} 0$ & $\mathrm{~K} 1$ & $\mathrm{~K} 2$ & $\mathrm{~K} 3$ \\
\hline $\mathrm{pH}$ & $3,7^{\mathrm{a}}$ & $3,6^{\mathrm{a}}$ & $3,6^{\mathrm{a}}$ & $3,5^{\mathrm{a}}$ \\
Acidez titulável \% & $1,2^{\mathrm{a}}$ & $1,3^{\mathrm{a}}$ & $1,4^{\mathrm{a}}$ & $1,5^{\mathrm{a}}$ \\
\hline
\end{tabular}

Médias com letras iguais na mesma linha indicam que não diferiram estatisticamente $(\mathrm{p}>0,05)$ pelo teste de Tukey.

K0-Natural (12\% de sacarose); K1 (12\% sacarose $+8 \%$ de polpa de goiaba); K2 (12\% sacarose $+10 \%$ de polpa de goiaba); K3 (12\% sacarose $+12 \%$ de polpa de goiaba). 
diferentes formulações de kefir após 7 dias de incubação e maturação diferindo apenas as concentrações de grãos e leite entre 1:10 e 1:5 respectivamente, obtendo valores de $\mathrm{pH}$ variando de 3,6 a 3,8 e acidez titulável de 1,4 a $2,7 \%$.

Os resultados das análises sensoriais mostraram diferença significativa $(\mathrm{p}<0,05)$ entre as formulações K0, K1 e K2 em relação a K3, para todos os atributos, exceto, a consistência. Pelas médias obtidas e levando-se em consideração ainda que o kefir não é um produto muito consumido pela população brasileira, as formulações analisadas apresentaram aceitação satisfatória, todavia, o fermentado de kefir saborizado com 8, 10 e $12 \%$ de polpa de goiaba, superou o fermentado de kefir natural (Tabela 2) em, basicamente, todos os atributos analisados, sugerindo que o incremento da concentração de polpa de goiaba de $8 \%$ para $12 \%$, tornou a cor mais atrativa e o sabor e aroma mais agradáveis, influenciando positivamente na aceitação.

Os resultados mais significativos, do ponto de vista da aceitação, dos atributos avaliados correspondem à cor, em uma variação de 7,5 a 8,5, observando-se melhores escores para as formulações com maior concentração de polpa de goiaba. O mesmo foi observado para o atributo sabor, com variação de 6,7 a 7,7 , e para o aroma, com médias variando de 6,7 a 7,4. O atributo consistência obteve as menores médias $(6,3$ a 6,5$)$, correspondendo ao termo hedônico "gostei ligeiramente", indicando que, apesar da adição de $2 \%$ de leite em pó não se observou correspondência positiva em relação a este atributo. Os resultados corroboram aos obtidos por Batista et al. (2014) que avaliando a aceitação sensorial de iogurte com $10 \%$ de polpa de banana e $10 \%$ de açúcar obtiveram médias de 7,03 ; 7,$08 ; 6,82 ; 7,04$ e 7,27 referentes aos atributos aparência, cor, aroma, consistência e sabor, respectivamente.

Nogueira et al. (2016) avaliaram a aceitação sensorial de kefir com duas concentrações distintas de polpa de açaí (30\% e $70 \%$ ) e verificaram que a concentração mais elevada de polpa também resultou em médias superiores para os atributos cor e sabor, entretanto a média da aceitação global foi inferior. Com base neste e nos resultados observados neste trabalho, sugere-se que há uma variação na aceitação de fermentado de kefir de acordo com a polpa utilizada para sua saborização.

Foi considerado como critério de aceitação global média $\geq 6,0$ (escala hedônica),

Tabela 2 - Intensidade de atributos sensoriais, expressa pela escala hedônica e intenção de compra de fermentado de kefir natural e saborizado com diferentes concentrações de polpa de goiaba

\begin{tabular}{ccccc}
\hline Atributos sensoriais & K0 & K1 & K2 & K3 \\
\hline Cor & $7,5^{\mathrm{c}}$ & $7,8^{\mathrm{c}}$ & $8,0^{\mathrm{b}}$ & $8,5^{\mathrm{a}}$ \\
Aroma & $6,7^{\mathrm{b}}$ & $7,0^{\mathrm{a}}$ & $7,1^{\mathrm{ab}}$ & $7,4^{\mathrm{a}}$ \\
Consistência & $6,5^{\mathrm{a}}$ & $6,3^{\mathrm{a}}$ & $6,4^{\mathrm{a}}$ & $6,5^{\mathrm{a}}$ \\
Sabor & $6,7^{\mathrm{b}}$ & $6,8^{\mathrm{b}}$ & $7.0^{\mathrm{b}}$ & $7,7^{\mathrm{a}}$ \\
Aceitação global & $6,7^{\mathrm{b}}$ & $6,9^{\mathrm{b}}$ & $7,0^{\mathrm{b}}$ & $7,7^{\mathrm{a}}$ \\
Intenção de compra & $3,4^{\mathrm{b}}$ & $3,3^{\mathrm{b}}$ & $3,6^{\mathrm{b}}$ & $4,3^{\mathrm{a}}$
\end{tabular}

K0-Natural (12\% de sacarose); K1 (12\% sacarose $+8 \%$ de polpa de goiaba); K2 (12\% sacarose $+10 \%$ de polpa de goiaba); K3 (12\% sacarose $+12 \%$ de polpa de goiaba).

Médias com letras iguais na mesma linha indicam que não diferiram estatisticamente $(\mathrm{p}>0,05)$ pelo teste de Tukey. 
conforme adotado por Araújo; Barbosa (2015) em análise de bebida láctea caprina saborizada com polpa de umbu, condizendo com os valores médios obtidos de 6,7, 6,9, 7,0 e 7,7 no presente trabalho, nas formulações K0, K1, $\mathrm{K} 2$ e K3, respectivamente. Estes resultados são satisfatórios, quando comparado ao observado por Puerari et al. (2012), que ao avaliarem após $48 \mathrm{~h}$ de fermentação a $25^{\circ} \mathrm{C}$, o kefir saborizado com polpa de cacau, utilizando escala hedônica de nove pontos, obtiveram média de aceitação correspondente a 4,68 .

Com relação à análise Multivariada aplicada, conforme observado na Figura 1, o primeiro componente principal (CP1) explicou $95,8 \%$ da variação dos dados, enquanto o segundo componente (CP2) explicou 4,1\%, totalizando $99,9 \%$ da variância existente entre as quatro formulações de kefir elaboradas (K0, K1, K2 e K3).

Observa-se que as formulações que obtiveram médias mais similares (K1 e K2), ocuparam regiões mais próximas no gráfico e são caracterizadas pelos vetores (atributos) que se apresentam mais próximos a elas, enquanto as formulações K0 e K3 estão localizadas em regiões distantes, entre os dois extremos. Neste tipo de representação gráfica, os vetores indicam que as variáveis são negativamente elevadas para esta, inferindo que, quanto maior a nota das mesmas, menor é o escore obtido. Deste modo, o escore inferior indica que o índice de aceitação é superior, o que comprova que a formulação K3 ( $12 \%$ sacarose $+12 \%$ de polpa de goiaba), sobressaiu sobre as demais, em relação aos quatro atributos avaliados, especialmente quando comparada a formulação K0 (kefir natural com $12 \%$ de sacarose).

Os resultados obtidos, especialmente para o fermentado de kefir saborizado com $12 \%$ de polpa de goiaba (K3), são satisfatórios quando comparados aos relatados por Martins et al. (2012) na avaliação da aceitação de fermentado de kefir adoçado com $10 \%$ de sacarose e saborizado com uma concentração elevada de polpa de morango (50\%) obtendo média de aceitação equivalente a 8,0 ("gostei muito"). Dalla Santa et al. (2008)

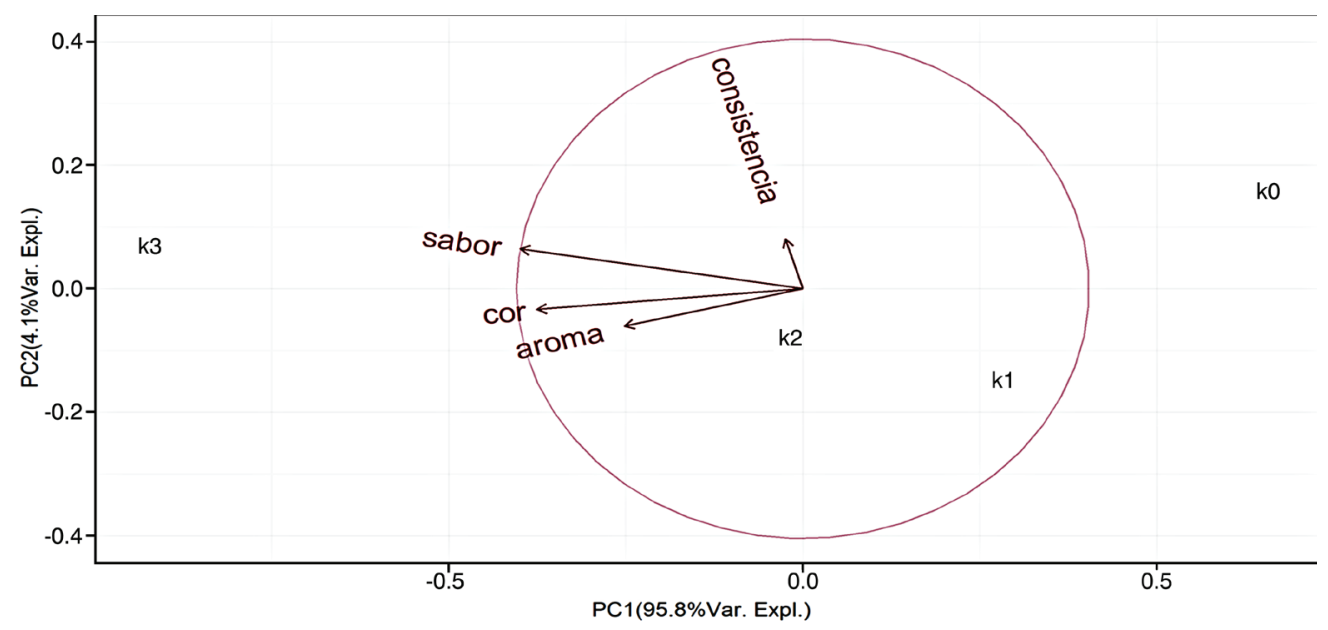

Figura 1 - Representação gráfica das formulações de kefir natural e saborizado com polpa de goiaba em relação aos dois componentes principais, pela interação entre os atributos cor, aroma, consistência e sabor. K0-Natural ( $12 \%$ de sacarose); K1 ( $12 \%$ sacarose $+8 \%$ de polpa de goiaba); K2 $(12 \%$ sacarose $+10 \%$ de polpa de goiaba); K3 (12\% sacarose $+12 \%$ de polpa de goiaba). 
avaliaram diferentes formulações de kefir, variando as concentrações de açúcar e polpa obtendo médias 7,0 e 6,8 para o kefir elaborado com 4 e $8 \%$ de polpa de ameixa, ambos adoçados com $11 \%$ de açúcar, e médias 6,4 e 7,2 para o kefir saborizado com $10 \%$ de polpa de morango, nas concentrações de 10\% e $12 \%$ de açúcar, respectivamente. Almeida et al. (2011) avaliaram uma bebida fermentada com grãos de kefir, adicionada de $8 \%$ de açúcar e observaram que uma porcentagem de $40 \%$ dos provadores atribuíram médias correspondentes a "gostei muito", inferindo uma atitude positiva de aceitação, enquanto que Montanuci et al. (2010) avaliaram a aceitação de kefir natural e obtiveram uma aceitação moderada, com média equivalente a 6,6 .

Quanto ao hábito de consumo, $96 \%$ dos julgadores afirmaram que nunca consumiram kefir natural e/ou saborizado, enquanto 4\% declararam um consumo moderado. Ressalta-se que não foi adicionado nenhum conservante e/ou estabilizante, corante e/ou aroma artificial ao kefir elaborado, indicando assim, uma avaliação positiva do mesmo. Dalla Santa et al. (2008) destacam a importância da aceitação do kefir na divulgação e incentivo do consumo, tendo em vista que a facilidade de elaboração do mesmo permite sua produção de forma artesanal, possibilitando o consumo também pela população carente.

A média de intenção de compra para o fermentado de kefir saborizado com $12 \%$ de polpa de goiaba foi 4,3 , indicando, que $86 \%$ dos julgadores "Provavelmente compraria" o produto, ratificando a boa aceitação atribuída e indicando que lograriam êxito no caso de uma produção comercial. Pelegrine et al. (2015) avaliaram a intenção de compra de duas formulações de iogurte de goiaba enriquecido com granola e aveia, obtendo uma porcentagem para o escore hedônico "certamente compraria" de $37,5 \%$ e $10 \%$, respectivamente. Para Guerra et al. (2011) médias entre 3,5 a 3,8 são satisfatórias. Nesse sentido, a formulação K2 se enquadra nessa classificação de intenção de compra.

\section{CONCLUSÕES}

O kefir elaborado com adição de $12 \%$ de polpa de goiaba pasteurizada apresentou uma aceitação e intenção de compra superior às demais formulações testadas, sendo, portanto, nas condições propostas, a concentração mais indicada para saborização deste tipo de leite fermentado.

\section{REFERÊNCIAS}

ALMEIDA, F. A. et al. Análise sensorial e microbiológica de kefir artesanal produzido a partir de leite de cabra e de leite de vaca. Revista do Instituto de Laticínios Cândido Tostes, v. 66, n. 378, p. 51-56, 2011.

Association Official Analytical Chemists (AOAC). Official Methods of Analysis, 12 ed., Washington, 2000.

ARAÚJO, N. G.; BARBOSA, F. F. Bebida láctea com leite caprino e soro caprino é alternativa para aproveitamento da polpa de umbu. Revista do Instituto de Laticínios Cândido Tostes, v. 70, p. 85-92, 2015.

BATISTA, D. V. S. et al. Aceitabilidade sensorial e caracterização físico-química do iogurte de banana cv. Terra. Enciclopédia Biosfera, v. 10, n. 18, p. 50-57, 2014.

BRASIL. Ministério da Agricultura e do Abastecimento. Resolução $n^{\circ} 5$, de 13 de novembro de 2000. Oficializa os "Padrões de Identidade e Qualidade (PIQ) de Leites Fermentados". Diário Oficial da República Federativa do Brasil, Brasília, 27 de novembro de 2000. Seção 1, p. 9-12. 
BRASIL. Ministério da Agricultura, Pecuária e Abastecimento. Instrução $\mathrm{n}^{\circ} 46$, de 23 de outubro de 2007. Regulamento Técnico de Identidade e Qualidade de Leites Fermentados. Diário Oficial da República Federativa do Brasil, Brasília, 23 de outubro de 2007. Seção $1,16 \mathrm{p}$.

DALLA SANTA, O. R. et al. Avaliação sensorial de kefir sabor ameixa e morango. Revista Brasileira de Agrociência, v. 14, n. 4-4, p. 77-85, 2008.

EVANGELISTA, R. M.; VIEITES, R. L. Avaliação da qualidade de polpa de goiaba congelada, comercializada na cidade de São Paulo. Segurança Alimentar e Nutricional, v. 13, n. 2, p. 76-81, 2006.

FERNANDES, A. G. et al. Avaliação sensorial de bebidas de goiaba adoçadas com diferentes agentes adoçantes. Ciência e Tecnologia de Alimentos, v. 29, n. 2, p. 358-364, 2009.

GUERRA, I. C. D. et al. Evaluation of goat mortadella prepared with different levels of fat and goat meat from discarded, Animals Small Ruminant Research, v. 98, n. 1-3, p. 59-63, 2011.

MARTINS, J. F. L. et al. Avaliação da adição do kefir em dieta hospitalar. Revista do Instituto de Laticínios Cândido Tostes, v. 67, n. 386, p. 13-19, 2012.

MEILGAARD, M.; CIVILLE, G. V.; CARR, B. T. Sensory Evaluation Techniques. London, CRP Press, Inc., 1991. 287 p.

MONTANUCI, F. D. et al. Caracterização sensorial e aceitação de Kefir adoçado integral e desnatado com inulina. Brazilian Journal of Food Technology, v. 19, n. 21, p. 79-90, 2010 .

NASCIMENTO, R. J. et al. Atividade antioxidante de extratos de resíduo agroindustrial de goiaba (Psidium guajava L.). Alimentos e Nutrição, v. 21, n. 2, p. 209-216, 2010.

NOGUEIRA, L. K. et al. Milk and açaí berry pulp improve sensorial acceptability of kefir fermented milk beverage, ActaAmazônica. v.46, n. 4, p. 417-424, 2016.

PELEGRINE, D. H. G. et al. Iogurte de goiaba enriquecido com cereais: correlação da textura com os parâmetros sensoriais. Revista de Ciência \& Tecnologia, v. 18, n. 36, p. 25-40, 2015.

PUERARI, C. et al. New cocoa pulp-based kefir beverages: Microbiological, chemical composition and sensory analysis. Food Research International, v. 48, n. 2, p. 634640, 2012.

SANTOS, M. R.; BASSO, C. Análise físico-química e sensorial de gelatina à base de quefir. Ciências da Saúde, v. 14, n. 1, p. 93-100, 2013.

SILVA, D. S. et al. Estabilidade de componentes bioativos do suco tropical de goiaba não adoçado obtido pelos processos de enchimento a quente e asséptico. Ciência e Tecnologia de Alimentos, v. 30, n. 1, p. 237-243, 2010.

WESCHENFELDER, S. et al. Caracterização físico-química e sensorial de kefir tradicional e derivados. Arquivo Brasileiro de Medicina Veterinária e Zootecnia, v. 63, n. 2, p. 473-480, 2011. 